1965 International Conference on Computational Linguistics

MACHINE TRANSLATION AND CONNECTEDNESS BETWEEN PHRASES

$$
\text { Karel ¿ U I I K }
$$

Mathematical Institute of Czechoslovak Academy of Sciences,ZZitná 25, Praha 1, Czechoslovakia

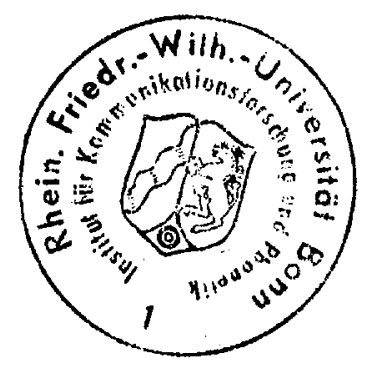




\section{Machine Translation.}

To translate a special text from one language into another means to construct to the given text in the first language, such a text in the second-one that has the same meaning (the same is told in it) like the given text。

The translation from a natural language $\mathrm{L}_{1}$ into a language $\mathrm{L}_{2}$ is such a function $F$ (in the more general case it is a manyvalued function) which assigns to any text $T$ in the language $L_{1}$ such a text $F(T)$ in the language $L_{2}$, that $F(T)$ has the same meaning like $T_{0}$. If we introduce a semantics (or interpretation) $M$ as a function (in general also many-valued) which to any expression $E$ of some language $L$ assigns its meaning $M[E]$ (compare [4]), it is possible to say that the function $F$ assigning the texts $F(T)$ from $I_{2}$ to the texts $T$ from $L_{1}$ is the translation only when

$$
M[T]=M[F(T)] \text { for any text } T \text { from } L_{1} \text {. }
$$

At the machine (or automatic) translation, the matter is, to define the function $F$ as a mechanizable procedure (i。e. algorithm) according to which an arbitrary starting text $\mathrm{T}$ in $\mathrm{L}$ is being succedingly modified till we get the translated text $F(T)$ fulfilling naturally ( $I$ ). The corresponding alsorithm can be finally programmed for a suitable computer.With respect to the used computer, the programne of the algorithm must not be too long, not even the wide range of memories must not be emploied and at last the translation must not take up too much of time. Usually it is required for the algorithm of a translation, to be the most effective. 


\section{Translation "Sentence by sentence".}

The translation $F$ is theoretically - as every function defined as a set of pairs $[T, F(T)]$ where $T$ runns through all texts in $I_{1}$. If we really had these pairs practically at disposal, we could use a trivial algorithm of the translation F: we should put in the memory of the computer all the pairs $\left[T, F(T)^{7}\right.$ and when being given the starting text $T$, we should find out in the memory the pair, in the first place of which $T$ would be situated, thus the pair $\left[\mathrm{T}, \mathrm{F}(\mathrm{T})_{-1}^{\top}\right.$, and the demanded translation $F(T)$ would be on the second place of this pair. This is, of course, not only funny but also impossible.

It seems to be funny because of the fact that to have practically at disposal the pairs $[\mathrm{T}, F(T)]$ it would mean to use live$1 \mathrm{y}$ translation and thus to translate all possible texts in advance. But the automation of translation signifies to exclude as much as possible the direct intervention of man out of the procseding of the translation and thus to sustitute a man by a machine. On that score, we do not possess practically the pairs $[\mathrm{T}, \mathrm{F}(\mathrm{T})]$.

It seems to be impossible because the texts $T$ are ton man (it would be possible to admit that infinitely many) and the pairs $[T, F(T)$.$] could not be included in any computer. On the other hand,$ it is necessary to admit that it concerus the algorithm, which is very simple (only to look up in the memory would take up too much of time).

The trivial algorithm being practically impossible, it is necessary to try to decompose long texts into parts and then to translata part by part. Naturally, it seems to be profitable, to treat sentences, that are in printed texts distinctly separated by points, as these parts. Thus, every text $T$ is a sequence 
of partial texts, i.e. sentences $S_{1}, S_{2}, \ldots, S_{k}$ so that we may write that $\left.T=\left[S_{1}, S_{2}, \ldots, S_{k}\right)\right]$. The function of the translation $F j . s$, of course, according to the asumption defined for all texts and thereby also for particular sentences $s_{1}, S_{2}, \ldots, s_{k}$ so that it is possible to construct a somposed text $\left[F\left(S_{1}\right), F\left(S_{2}\right), \ldots, F\left(S_{k}\right)\right]$ from the translations of these sentences $F\left(S_{1}\right), F\left(S_{2}\right), \ldots, F\left(S_{k}\right)$, that are some partial texts in $L_{2}$. At the same time, the translations of sentences follow in the same seciuence as did the starting sontences in the text $T_{0}$. It may happen - and we should sure welcome it, if it were always - that it holds

$$
F\left(S_{1} \circ S_{2} \ldots S_{k}\right)=\left[F\left(S_{1}\right) \circ F\left(S_{2}\right) \ldots F\left(S_{k}\right)\right]
$$

or at least the weaker condition

$$
M\left[F\left(S_{1} \circ S_{2} \ldots S_{k}\right)\right]=M\left[\left(F\left(S_{1}\right) \circ F\left(S_{2}\right) \ldots M\left(S_{k}\right)\right]\right.
$$

rom (2) there follows (3) but in no way the contrary. For the translation the condition (3) is sufficient. It might, namely, happen that we translate the text $T$, as a whole, differentIy than when translating it succedingly in parts $s_{1}, s_{2}, \ldots, s_{k}$, so that (2) does not hold, but despite this (3) holds.

In the condition (2) and similarly the condition (3) were fulfilled for any text $T=\left(S_{1} \circ S_{2} \ldots S_{k}\right)$ in $L_{1}$, it would signify that it was always possible to translate single sentences of the text quite independently each of another. It is probably not true. Sometimes, it is necessary to know, how the sentence $S_{I}$ was translated, when we want to translate correctly the sentence $S_{2}$, becau- 
se regularly both sentences are connected as to the contents, and not always this connection is expressed by syntactical means. In addition, sometimes it is necessary to translate too long sentences from $L_{1}$ as two or more sentences from $\mathrm{L}_{2}$ and then not even the sequence is possible to be defined in advance.

In spite of this, the condition (2) or at least (3) is the basic asumption for any translation "sentence by sentence" and most part of translation belongs to such a type of contemporary translations. To be competent to accept the asumption (2) or (3) it sufficies to confine oneself to some texts only, and the texts not fulfilling this assumption are necessary to be adapted before the translation in ordar to make them able to fulfill it. It is not clear, of course, how to find it out at the given text, before starting the translation.

ihe asumption (3) stands for nothing else than

(4) $M(T)=M\left[F\left(S_{1}\right) \cdot F\left(S_{2}\right) \ldots F\left(S_{k}\right)\right]$ for any text $T=\left[S_{1} \cdot S_{2} \ldots S_{k}\right]$ and hence the substantial simplifying of the definition of translation can to be seen. It suffices, namely, to suppose that it is necessary to define only a partial function $F^{*}$ of the function $F$, that is defined for arbitrary sentences in $L_{1}$ only (on no account for arbitrary texts when the sentences are a special case of simple texts). Thus, there holds $F^{*}(S)=F(S)$ for every sentence from $L_{1}$ and out of (2) there follows

(5)

$$
F\left(S_{1} \cdot S_{2} \ldots S_{k}\right)=\left[F^{*}\left(S_{1}\right) \cdot F^{*}\left(S_{2}\right) \ldots F^{*}\left(S_{2}\right)\right]
$$


so that in fact we are able to cope with the function $F^{*}$ when translating the texts.

Similarly, like at the function $F$, it is possible at the function too, to try to give a trivial algorithm making full use of all pairs $\left[S, F^{*}(S)\right]$. But the situation improves only a little. It wold be again necessary to translate all the sentences in advance and even these are still too many, so that all preceding reasons remain valid, what means, that it is necessars to try to decompose even the sentences in parts, and to translate the sentences in parts, too.

\section{Translation "word by word".}

By the decomposition of the translation into sentences there were no difficulties because in printed texs this decomposition into sentences was just ready, and according to the syntanctical means it was possible to decompose its parts。

of the same simplicity and uniqueness is the decomposition of the sentence $S$ in its single words $W_{1}, W_{2}, \ldots, w_{k}$ separated by interspaces, so that it is possible to write $s=\left(W_{1} W_{2} \ldots W_{k}\right)$ like at the text.

Besides, the linguists have constructed, a long time ago, a binary translative dictionary from the language $L_{I}$ into the language $I_{2}$. This dictionary is, in fact, defined as a set of the pair of words, the first-one from $L_{1}$ and the second-one from $\mathrm{I}_{2}$ having the same meaningoIf we denote by $f$ the translation from $L_{1}$ into $L_{2}$, where $f$ is again generally many-valued 
function (thanks to the homonymy of words) $f$ evidently fulfils the condition (in details ses [ 3$]$ )

$$
M[f(W)]=M(W) \text { for every Wrom } L_{1} \text {. }
$$

In whatever way the trivial algorithm of the translation of texts and sentences was funny and impossible, this algorithm is in case of the translation of words not only possible, but also it is used from time to time by living translators. At most machine translations, there is really choosen for the algorithm of the translation $f$ the just mentioned trivial algorithm, i.e. into the memory of a computer there are input all pairs $W, f(W)$ and the most tedious procedure is - how it wes said- to look up in the memory and to compare.

But it is not necessary to use this trivial al corithm。 It is possible to construct a sequential automaton and thus to construct also a corresponding technical apparatus which will realize the function $f$, ioe o if there enters on its input the word $W$ as a sequence of letters $l_{1} l_{2} \cdots \circ I_{k}$, which is eventually prolongated by the means of several help-symbols (compare [5]) we get on output again the sequence of letters $1_{1}^{*} 1_{2}^{*} \ldots 1_{k}^{*}$, that eventually starts with several help-symbols and simultaneously there holds that

$$
f\left(1_{1} I_{2} \ldots I_{j}\right)=1_{i}^{*} 1_{i}^{*} \ldots 1_{k}^{*} \text {, when } w=1_{1} 1_{2} \ldots 1_{j}, j \leq k \text {. }
$$

There is a question, whether there is not possible, when using this automaton, to shorten the time necessary for translating, when evidently all lost times can be excluded at looking up in the input dictionary. 
Taking no account to the fact, in what way the translation of words $f$ is given, one may ask, whether for the sentence $s=\left(W_{1} W_{2} \ldots W_{k}\right)$ there holds

(8) $F^{*}(S)=\left[f\left(W_{1}\right) f\left(W_{2}\right) f\left(W_{2}\right) \ldots f\left(W_{k}\right)\right]$,

which is the similar condition to the condition (2) for the translation of texts.

It. is known that this condition holds nearly never for most natural languages, becanse the translation of words $f$ is the translation of words in basic form only, whereas by the decomposition of the sentence $W_{1}, W_{2}, \ldots W_{k}$ are in various word-forms. To put it differently:the function $f$ respects only the lexical meaning of words but does not take into consideration morpholosical questions.

However even in the case, the function $f$ could be prolongated from basic forms on other form of words-what, of course, need not be possible-or on the contrany, if we adapted to the basic form single words $w_{i}$ in the decomposition of the sentence, yet despite this all - evon under these suppositions - the condition (8) would be fulfilled in the rase only, that there are concerned two languazes $\mathrm{L}_{1}$ and $\mathrm{L}_{2}$ that are very strongly cognate, or two dialects of the same language, or in the case $L_{1}$ and $L_{2}$ are not cognate, the considered sentence $s$ must be very simple.

The translation fulfilling the condition analogous to the condition (8) may be called the translation "word bji word". But unfortunately it is known that such a translation is im- 
possible in natural languases, although it would be very advantageous and simple。

It does not mean, of course, that the decomposition of the sentence into words cannot be used; it is too fine and therefore it is necessary to decompose the sentence in another way, at all events in such a way that single parts will contain more than one word (and we suppose, under tacit consent, that the words have full meaning, not only being help-words with the erammer meaning) and that these parts need not be sentences. Before introducing these parts, it is necessary to take into consideration various necessary morphological and grammatical statements, and theroby to adapt properly the condition $(\varepsilon)$, too, wher a there were no differences between the basic word-form or the mere stem of the word and its various possible forms. 
4. Syntactical and senantical characteristics of words.

First of all we may suppose that, to every word-form (shape) which appeared in the decomposition of some sentence into woras, we are able to define its basic form or stem if (the function $f$ refers just to these basic forms) and its characteristic $c=\left(x^{1}, x^{2}, \ldots, x^{n}\right)$, where $n$ is according to the need a sufficiently great integer and single $x^{j}$ are some gramatical morphological and eventually even other data referring to the form W. For example $x^{1}$ can be the datum on word-kind, $x^{2}$ the datum on case, $x^{3}$ on gender, $x^{4}$ on number, $x^{5}$ on person, $x^{6}$ on time, $x^{7}$ on mood a.s.o.

Naturally we also assume that on the contrary, if it is given the basic form $w$ and prescribed (of course admissible) the characteristic $c$, it is easy to define the starting form $i$. Thus, we suppose that there are given functions $h$ ano $h^{-1}$ such that $h(W)=(w, c)$ and $h^{-1}(w, c)=W$ in each of considered languares (in $L_{1}$ and $I_{2}$ it will be functions $h_{1}$ and $h_{2}$ ).

Although it is well known that the sentence and its translation need not have the same number of words, this demand is not far from truth when we pay attention only to the full-meaning words. Iet us consider, however, such sentences $S$ which fulfil this demand (this is the supposition for making the coment easy) i.e. if it is $S=\left(w_{1} w_{2} \ldots W_{K}\right)$ then

$$
F^{*}\left(W_{I} W_{2} \cdots W_{K}\right)=\left(\bar{W}_{I} \bar{W}_{2} \ldots \bar{W}_{K}\right),
$$


where evidently $\bar{W}_{1}$ are the words of the decomposition of the sentence $F^{*}(S)$. The task is, how to get the chain $\left(\bar{W}_{1} \bar{W}_{2} \ldots \bar{W}_{K}\right)$ from the given chain $\left(W_{I} w_{2} \ldots W_{K}\right)$ and we know already that it is not possible to get it by means of the translation word by word according to (8) directly.

When we use a function $h_{1}$ we get from any word-form ' $W_{i}$ its basic form $w_{i}$ and characteristic $c_{i}$, namely, $h_{1}\left(w_{i}\right)=$ $=\left(w_{i}, c_{i}\right)$ and thus we can differentiate the data $\left(W_{I}, W_{2}, \ldots, W_{K}\right)$ and data $\left(c_{1}, c_{2}, \ldots, c_{K}\right)$.

It is similar with the translated sentence $\left(\bar{W}_{I} \bar{W}_{2} \ldots \bar{W}_{K}\right)$ when one uses the function $h_{2}$. Again we are able to discern data $\left(\bar{W}_{I}, \bar{W}_{2}, \ldots, \bar{w}_{K}\right)$ and data $\left(\bar{c}_{1}, \bar{c}_{2}, \ldots, \bar{c}_{\mathrm{K}}\right)$ when evidently. $h_{2}\left(\bar{w}_{i}\right)=\left(\bar{w}_{i}, \bar{c}_{i}\right)$.

Now, it is clear that instead of the condition (8) ought to be the condition

$$
\bar{w}_{i}=f\left(w_{i}\right) \text { for } \quad i=1,2, \ldots, k
$$

because here $f$ is really used for basic forms of words. Then the translation fulfilling (10) is in fact the translation "word by word" but only in the respect of the meaning of word, being far from complete translation. There is missing the proceeding, how to get from the starting characteristic $\left(c_{1}, c_{2}, \ldots, c_{K}\right)$ characteristic $\left(\bar{c}_{1}, \bar{c}_{2}, \ldots, \bar{c}_{K}\right)$. And just here there is impossible except for the most simple example - to find such a function $g$ in order to hold

$$
\bar{c}_{i}=g\left(c_{i}\right) \text { for } i=1,2, \ldots, k \text {. }
$$


If it were the case, or in these cases for which the function is could be fourd, the translation would be easy, because it would eviaently hold

$$
\bar{w}_{i}=h_{2}^{-1}\left[f\left(w_{i}\right), g\left(c_{i}\right)\right] \text { for } i=1,2, \ldots, k
$$

where, of course, $h_{1}\left(w_{1}\right)=\left(w_{i}, c_{i}\right)$ for $i=1,2, \ldots, k$.

As it is impossible to translate one characteristic after another, it is necessary to use instead of the supposed (but in general not existing) function $g$, the more complicated function c. This function will not define single characteristics $\bar{c}_{i}$ in dependence on the sole characteristic $c_{i}$ as it was to be in (II), but in dependence on all characteristics $\left(c_{1}, c_{2}, \ldots, c_{K}\right)$ so that it nay be written analogically to ( 9 )

$$
G\left(c_{1}, c_{2}, \ldots, c_{K}\right)=\left(\bar{c}_{1}, \bar{c}_{2}, \ldots, \bar{c}_{K}\right)
$$

where, properiy, would be necessary to differentiate functions $G_{1}, G_{2}, G_{K}$ and put $\bar{c}_{i}=G_{i}\left(c_{1}, c_{2}, \ldots, c_{K}\right)$ for $i=1,2, \ldots k$. Whereas the condition (10) has been fulfilled quite frequently (especially at' simple sentences and above all when we weaken it by adintting the changed the ordering of words $\bar{W}_{i}$ in comparison with words $\left.w_{i}\right)$, i.e. it is often possible to translate word by wora as for the meanings of single words, the condition (II) has nearly never been fulfilled. It can be understood, because in the respect of meaning the languages do not differ as a natter of fact and this matters in (10), while morpholobically and eventually even sranratically single languages differ very strongly and these racts matters in (II). 
From this also follows that difficulties of impossibility of the translation word by word according to (8) are - for the differentiation of the meaning anc characteristic - due to the characteristic and not to the mearings. This fact has a considerable heuristic import. It is, nanely, evident that the suitable parts into which we want to decoupose the sentences are to be found with respect to their significance and not with respect to their syntactical or even morpholosical properties. In this case, namely, these parts will be found at the same tire in all. languages even is having been expressea in different languages by different syntactical an morpholosical means. It is naturally self-evident that between the significance of considerea parts and their syntactical expressions are close connections (see [2]).

Because of this, it is necessary to introduce, besiajes the wentioned characteristics some others nore, naxely, logical and semantical that will be common for all languazes and will be ouite independent of the syntax of languages. And just this condition is fulfilled by the logical and semantical questions.

Under the logical characteristics of woras we understand data on the fact whether and whet logical corjuctions or other lopical mears (as ouantors or negetions) are by these mords expressed. These lacts are known from the lobical analysis of sentences worked out by $R$. Carnap.

By the cenmantical characteristics of words we understana data on the fact whether the given word (we suppose word with lull reaning, in no way gramatical or Iogicul woras) plays the role of indiviaual constant, or variable i.e. whether it derines a certain object 
(here the term object is used in the wide sense of the term) or an arbitrary-one with certain propertied, or plays the role of one-placed predicat, i.e. denotes some property, or of two-placed predicat, $i . e$. denotes two-member relation, or in general n-placed preaicat, i.e. denotes n-figurea relation.

The situation is not so hopelessly complicated as it would seem at the first sight. For instance, the individual constants even the variables are only substantives while verbs are always predicats one-. two- three- even more placed, according to the smaller or greater number of their objects. Adjectives are always one-placed predicate a.s.0.

Besides the mentioned - and in the logic current - it is necessary to consider as semantical characteristics data on time and place and probably not yet cuite distinctly defined data referring to the conditions under which the situation is being described (here belons sone adverbial modifier).

Thus, we suppose that we know the function $k$ (analogously like $h$ ) which to any word-form $W$ ascigns its logical and serantical characteristic $a$, thus, $k(W)=a$, while again $\mathrm{d}=\left(\mathrm{y}^{\mathrm{I}}, \mathrm{y}^{2}, \ldots, \mathrm{y}^{\mathrm{K}}\right)$ where e.e. $\mathrm{y}^{I}$ is a datum, whether the word $W$ is the logical functor and of what kind, $y^{2}$ is a datum whether the word $y$ is preuicate ana how many-placed, $y^{3}$ a alum of what kind the preaicate is, $y^{4}$ whether there is in the definition of time and of what kind, $y^{5}$ whether $w$ is the acrinition of place and of what type, $y^{6}$ a datum on the special derinition of the concition a.s.o. 


\section{CuIik 14}

\section{Primitive phrases.}

With respect to the semantical characteristics of woras it is possible and quite natural - similarly as in the predicate-logic to take as important in a sentence such phrases (i.e. their parts) that include always one worci playing the role of $n$-placec precicate so called basic predicate - in this phrase (while phrases contain $n+1$ words) and the other words (just in the numiver $n$ ) play the role of individual constants or variajles being placed on single places (positions) of the considered preaicate. In accordance with the mentioned it referrs, in this phrase, to the denotation of the n-membered relation and to the denotation of all $n$ objects that are mutual in this relation. Thus, every such phrase is, in pact, a certain statement or a definition on the situation. As it is the analogy of the primitive formula in the nathematical logic (e.j. $P\left(a_{1}, a_{2}, a_{3}\right)$ is the primitive fomula when we know that $?$ is a three-figured predicate, that $x_{1}, x_{2}, x_{j}$ are indivicual constents placed on their three places, and that the mentioned record says that the objects denoted by these constants are in relation denoted by the predicate $P$ ) we call such a phrase the primitive phrasc.

Simultaneously, it is imnediately evident that the prinitive phrase need not have the grantatical form of a sentence, and in most cases it really does not have it. It has the form of a sentence just when its preaicate is a verb anc when this verb has not the Eramatical gon of a gerodna on a participle. For instance "a man reads a book" is the wrimitive phrase in the form of a granatical sentence (nure Evidently "reacs" plays the role of two-ribured precicate), but the primitive phrase "a man reading book" on na man who 
is reading a book" has not the form of a sentence even if having the same neaning like the preceeding phrase, because both express the sane fact. Some other types of primitive phrases are e.e. these "very good" where "very" is a one-placed predicate and on the place of it stands "good" (although in another primitive phrase "good. book" is good itself a one-placed predicate), or "reads quickly", where a one-placed predicate is quickly a.s.o.

From these examples there follows that primitive phrases correspond with primitive fomulas in the predicate logic of higher order. In the phrase "man is mortal" there is evidently concealed the universal quantor "every" so that this phrase has the same meaning like "every man is mortal" and thereby to not a primitive phrase but a composed-one.

With regard to the syntactical side, the primitive phrases differentiate on the basis of the characteristics of single words. For instance, the sequence of word-characteristics $\left(c_{1}, c_{2}, c_{3}\right)$ where $c_{1}, c_{2}, c_{3}$ are such that $c_{1}$ denotes a substantive in the first case, $c_{2}$ denotes a transitive verb, $c_{1}$ and $c_{2}$ simultaneously coinciding in their components as for the gender and number, and at last $c_{3}$ sicnifies that it referrs to a substantive in the fourth case, when, in addition, the range of characteristics sets the future word-order, is the characteristic of the primitive phrase "a man reaủs a book" (when an indefinite article is a consiaered not to be self-contained word, and we assign it always to the word suceeding it immediately). If we, namely, made full use of the function $h$ we would get $h$ [a man $]=\left[\operatorname{man}, c_{\mathbb{l i}}\right]$, in [reacis $]=\left[\mathrm{read}, c_{R}\right]$ and $h[a b o o k]=\left[\right.$ book, $\left.c_{B}\right]$, and 
simultaneously it couls sure hold $c_{\mathbb{M}}=c_{1}, c_{R}=c_{2}$ and $c_{\mathbb{Z}}=c_{3}$. The considered language is, besides its word-store put down in the dictionary, characterized also by the list of all characteristics of primitive phrases. That is what we shall suppose at any of the considered languages.

If we use for the considerea primitive phrases. That is what we shall suppose at any of the considerec languages.

Ir we use for the considered primitive phrase "a man reads a book" the function $k$, we get some semantical word-characteristics $a_{\mathbb{N}}, d_{\mathbb{R}}$ and $d_{B}$, and one of then will be especially distinguished as a basic predicate of the considered type of the primitive phrase. In our case it is $a_{R}$ and for illustration we shall come to an agreement that this basic predicate and other semantical characteristics will be put down in the same way like it is aone in the preaicate losic, namely $a_{R}\left(a_{M i}, a_{B}\right)$. Let us call this entry the semantical characteristic of the considered phrase and also the senantical characteristic corresponding with the syntactical characteristic $\left(c_{M}, c_{R}, c_{E}\right)$ of the considered phrase. inalozous agreements are to be made even with respect to other conponents of the senantical characteristic, particularly for the definition of tirite, place and other conditions.

At the same tine, the semantical characteristic comprises these facts: $d_{R}$ a daturn that it refesrs to the two-fisured preaicate (eventually specialized by the denotation of some activity), on the first place of which is just the word, with the semaniical characteristic $d_{M}$ (e.g. with a supplenent "agens") and 
the second place of which corresponds with the semantical characteristic $d_{B}$ (eventually with supplement "patiens"). Simultaneously, the semantical characteristics may be eventually complemented with further data, when it turns out to be suitable. It is irportant only, that there are to be data (as it is mentioned in the parentheses) that referr to the meaning and that are common for all languages.

By the semantival characteristic $d_{R}\left(d_{W /}, d_{B}\right)$ there is put down, in the basic semantical categories, just what we want to express (By that tire the basic foms Read, Man, Book are failine; these are possible to be chosen differently) while the corresponaing syntactical characteristic $\left(c_{M}, c_{R}, c_{D}\right)$ puts down how to express it.

Now, the way is evident, how to translate primitive phrases from the langlage $I_{1}$ into $I_{2}$. There is inportant that we suppose that whatever can be expressei in $I_{I}$, can be expressed even in $I_{2}$ what is the basic supposition on the possibility of translatins. From this there follows for the function of the translation of. words $f$ that for every basic form $W$ from $I$ there exists $f(W)$ in $I_{2}$, and that for every semantical characteristic $a_{1}\left(d_{2}, a_{3}, \ldots, a_{n}\right)$ corresponding with the syntactical characteristic $\left(c_{i_{1}}, c_{i_{2}}, \ldots, c_{i_{n}}\right)$ in $I_{1}$ thete exists the syntactical characteristic, correspondins with it $\left(c_{j_{1}}, c_{j_{2}}, \ldots, c_{j_{n}}\right)$ in $\mathrm{I}_{2}$ and just this-one (of course they may be several) will be declarea to be the trabslation of the corresponding syntactical characteristic from $I_{1}$. Thereby a further function $\varphi$ is defined (eventually a many-valued) for which there holds that 
(I4) $\varphi\left(c_{i_{1}}, c_{i_{2}}, \ldots, c_{i_{n}}\right)^{I}=\left(c_{j_{1}}, c_{j_{2}}, \ldots, c_{j_{n}}\right)^{2}$, when $(\ldots . .)^{I},(\ldots \ldots)^{2}$ have the sure semantical characteristic. Now, if it is given a prinitive phrase $\left(w_{1}, w_{2}, \ldots, w_{n}\right)^{l}$ in $I_{1}$ we use first the function $h$ and we get $h\left[\left(W_{1}\right)\right]=$ $=\left[w_{i}, c_{i}\right]$ for $i=I, 2, \ldots, n$, and thus $i t s$ syntactical characteristic $\left(c_{1}, c_{2}, \ldots, c_{n}\right)^{I}$ but now $\varphi\left(c_{1}, c_{2}, \ldots, c_{n}\right)^{I}=$ $=\left(c_{j_{1}}, c_{j_{2}}, \cdots, c_{j_{n}}\right)^{2}$ so that

(I5) $\quad F^{*}\left(W_{1} W_{2} \cdots W_{n}\right)=\left(h_{2}^{-1}\left[f\left(w_{j_{1}}\right), c_{j_{1}}\right] h_{2}^{-1}\left[f\left(w_{j_{2}}\right), c_{j_{2}}\right] \ldots\right.$

$$
\left.\cdots h^{-1}\left[f\left(w_{j_{n}}\right), c_{j_{n}}\right]\right)
$$

and this is, in fact the needed weakening of the concition (12).

It is evident that the primitive prrase from $\mathrm{I}_{2}$ on the richthand side of the equation (15) has reaily the same meaning as the primitie phrase on the lert-hand of the equation. As for the meanine of sinele words, this is guaranteed by the function $f$ and as for the meaning of the whole phrase, it is guaranteed by the function $\varphi$, that fulfilis (14) that here in a special case plays the role of the function $G$, because (14) and (13) are identical. 


\section{Compound phrases.}

The composing of two or more primitive phrases in compound phrases can be performed by the usual logical means (e.E. by neans of logical conjunctions "even" or "if,...then..." eventually of other analogous conjunctions like "but" and similar, or by means of negation and quantors) in a well-kncwn way, or can be perfomed by the fure lineuistic expressive means. Both these kinde may be arbitrarily interchanged by the successive composing.

Similtaneously, it is decisive that the composing of single primitive phrases corresponds with the composing of their syntactical and, of course, semantical characteristicso Besides, therz are mostly composed such two phrases that have some word in common, or where some word is repeated. this fact is necessary to be distinguished especially by composing the corresponding rharacterictics, or - what is in substance the same- it is necessary to join to ary word-characteristic $c$ the symbol expressing a variable for the basic forms of word, so that we shall write ${ } \mathrm{x}, c_{1}$ ] where we can put for $X$ the real basic forms of words.

The composing of primitive phrases in compound phrases belong to the field of the synthesis of phrases. If we, for instance, want to say that some man reads a book and simultaneously that he reads quickly and, in addition, that this bock is cood and even very good, we can express it in the following cnmpund phrsses $P=$ (which is grammetically the form of the sentence) "man reads quickly a very good book". The syntax of this phrnse is not evidently expressed bj the logical meanso 


\section{x.2] 20}

In the consilered case the following primitive phrases are concerned: $P_{1}=$ " a ma reads a book, " $P_{2}=$ " reads guickly", $\mathrm{P}_{3}=$ "good book" and $\mathrm{P}_{4}=$ "very good". From these phrases the compound phrase is put togethor. If we use the function $h$ for single words of the considered phrase, we get successively $\mathrm{h}[\mathrm{a} \operatorname{man}]=\left[\operatorname{man}, c_{1}\right], \mathrm{h}[$ quickly $]=\left[\right.$ quick, $\left.c_{2}\right], \mathrm{h}[$ reads $]=\left[\mathrm{r} \Leftarrow \mathrm{ad}, c_{3}\right] \ldots$, ,,,,$h[$ a book $]=\left[\right.$ book, $\left.c_{6}\right]$, where $c_{1}, c_{2}, \ldots, c_{6}$ are the corresponding syntactical characteristics. Analogously when we use the fun ction $k$ we get the semantical characteristics of sincle wordso

The syntactical charcicteristics of single (separate) primitive phrases $P_{1}, P_{2}, P_{3}, P_{4}$ are successively $C_{1}, C_{2}, C_{3}, C_{4}$, where $c_{1}=\left(\left[w_{1}, c_{1}\right],\left[w_{3}, c_{3}\right],\left[w_{6}, c_{6}\right], c_{2}=\left[w_{2}, c_{2}\right],\left[w_{3}, c_{3}\right]\right)$ $c_{3}=\left(\left[w_{5}, c_{5}\right],\left[W_{6}, c_{6}\right]\right)$ and $c_{4}=\left[w_{4}, c_{4}\right],\left[w_{5}, c_{5}\right]$ ). At the same time, thare is very important that some variables $N_{i}$ occur simultaneously in two primitive phrases. Thereby is, namely, expressed the circumstence that by these two phraes is told something of the same fact and just this circumstance plays the decisive role at stating the constents-connection among more phrases。

If we started from the Eiven phrase " a man quickly reads a very good book" we would find the mentioned four primitive phrases as follnws: first we would use for single words the function $h$ and $k$ and then we would find for every word of the mentioned phrase, which can be the basic predicate of some primitive phrase (it can be found out of its semnatical charecteristics and by the semantical characteristics of the primitive phraes) further words beloncing to it in a certain priritive phrase, ioe, which take places of the considered predicate and this sccurs only 
in comparison of the syntactical characteristics of words frcm the given phrase with the syntactical characteristics of a certain investigated primitive phrase.

For instance, in our case, if we have found out according to the semnatical characteristic that the word "reads" is tivoplaced predicate, we would find out the syntactical characteristics of such primitive phraes, the besic word of which was just the two-placed predicate. Then we have kncwn what syntactical characteristics of words and - as far as the word-order is concerned - where there are to be found, so that we find out whother the investigated primitive phrases are in the given phrase included. When finishing it for all these words, we shall find it successively for all primitive phrases that in the given phrase are comprised.

In such a way is, namely, depicted the analysis of the compound phrase, not composed by the locical means. If thare are used the lorical means, then the given phrase is decomposed like in the logic。

Rut it is necessary to mention in adrition, that for the eccnomy-reasons and for saving the number of syntactical characteristics of the primitive phrases, it is convenient to work often with incomplete characteristics only. The question is, whether we shall include two primitive phrases" a man reads a book" and "the man read a book" into one (incomplete) syntectical characteristic, or into two different and naturally complete -ones. The incompleteness will consist in the feiling fact on number (and similarly it would be in other phrees with data on ender and case), but naturally there would not fajl 
the datum on coincidence in number between "a man" and "reads or "the man" and "read" because this fact will be just decisive for the incomplete characteristics.

The possibility of the use of incomplete syntactical characteristics by the synthesis is, of course, also evident. If we want to make the whole synthesis of the compound phrase independent on proper meanings of single words, then we can give in the syntactical characteristics neither the gender nor the number, becsuse both of them are defined differently no sconer than by the choice of the basic form because in many cases genders are steadily fixed). But even here it is not the matter of principle but the matter of effectivity.

\section{Semantical dependence and connectedness.}

As, according to the supposition, there is denoted in every primitve phrase its basic prodicate which always stands in front of parantheses in its semantical characteristic (for instance at $Q(x, y) \quad Q$ is the basic predicate) it is possible to define the semantical dependence among the words of the prinitive phrase by the demand that the basic predicate always depends on all other woras that occur in the phrase, i.e. on its arguments (e.g. a depends on $x$ and on $y$ ). Just so justified would be the definition that, on the contrary, all argunents depend on the basic predicate.

If we demonstrate this semantical dependence on a diasram, we always draw the connectine line, provided with an arrow-head, directing from an angument to a basic predicate. At the same time, 
of course, according to the position of separate words - if they are more to the left or to the right - we discern always the word-order. Four prinitive phrases from the preceeding paragraph are deomstrated in the following diagramm:

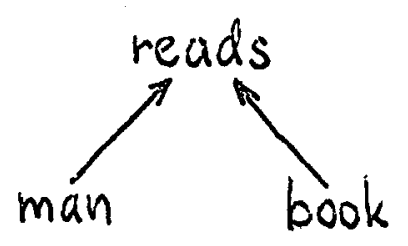

$P_{1}$

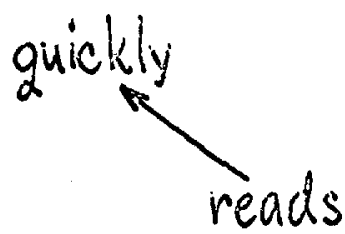

$P_{2}$

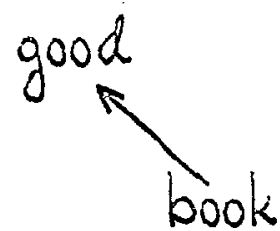

$P_{3}$

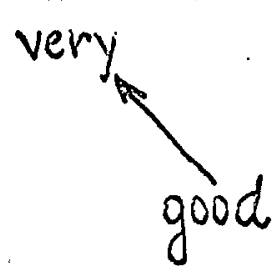

$P_{4}$

We say that the semantical dependence is concerned because this relation among the basic predicate and its arguments, expressed just by the theorem that objects denoted by the arguments are in relation defined by the basic predicate, is quite initial definition referring evidently to the reality. The semantical dependence does not refer to anything else than to the fact of telling somethins of something (on the mathematical level the fact of telling $Q(x, y)$ can be transferred only on the basic relation of the aodherence to the set) when one passes from the predicate $\&$ to the binary relation $\varepsilon^{*}$ and puts down $\left.(x, y) \in q^{*}\right)$.

We say further that in the primitive phrase the basic predicate is directly connected with any of its arguments, i.e. two words of the primitive phrase cohere together when either the first depends on the second, or the second on the first. When illustrating the direct connectedness we can use the same diagramm like when illustrating the dependence, but we do not pay attention to arrow-heads. Thus, evidently in. $\mathrm{P}$ "man" is directly connected 
with "reads" but is not connected with "books" a.s.o.

But if there is given the phrase and if we have found all its primitive phrases, then it often happens that some word of the given phrase will occur (come up) in several priritive phrases. This evidently signifies that we are justified to identify the repeated words (naturally not always)occurring in difierent priritive phrases of the same sentence, and that is what we do by illustrating it on diagramm. In the considered case of the sentence "a man reads quickly a very good book" we get on to the diagran:

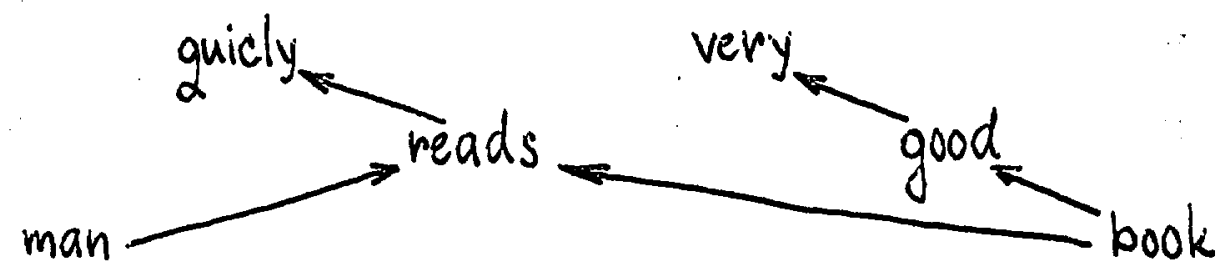

P

In this way, there are defined the semantical dependences among single words of the given sentence. The connecteaness (no more direct) anong single words of the sentence will be introduced in the way, common in the theory of graphes, namely, the words $x$ is connected with the word $y$ in the phrase $P$ when there exists a finite sequence of the words of the sentence $P, z_{I}, z_{2}, \ldots, z_{K}$ such that $z_{I}=x, z_{K}=y$ and that $z_{i}$ and $z_{i+1}$ immediately cohere together for any $i=1,2, \ldots, k-1$.

Finally, if there is given the whole text consisting of single phrases, to which we have found their diagramms of the semantical dependence or connectedness, then often would happen that in the succeiing phrases the same objects are spoken of, like in the preceeding-ones. Sometimes, this fact is distinctly expressed 
by the referrin means (there are e.g. pronouns, derinite articles and simiar), but sometimes these are concealed and in this case it will be necessary to complete the text (or not to admit such a text at all). If there are everywhere the referring means expressed, they are possible to be usei for further identification of the words of single diagramins for separate phrases (analogously as it was mentioned at the primitive phrases), and thereby to get the diagramms of the senantical dependence, eventually even the dependence for the whole text.

In the case of the whole-text-diagramm two cases are possible: either there is a connected graph and then we say that the connectea text is concernec, or this graph is disconnectea and then we say that the text is disconnectea. But, any disconnected text splits, in a natural way, into its connected components and it is evident that it will be possible to transiate these components independently on themselves because they do not cohere together semantically).

Therefore we can concern only a connected context T. According to the section $2 \mathrm{~T}=\left(S_{1} \cdot S_{2} \cdot \ldots S_{k}\right)$, where $S_{i}$ are sentences and we remind that the condition (2) resp.(5) is not allways satisfied, because e.g. sometimes it is necessary to know, how the sentence $S_{1}$ was translated, when we want to translate correctly the sentence $S_{2}$. But now it is simple to see that there is exactely one word $w^{2}$ in $S_{1}$ and $w^{2}$ in $S_{2}$ such that $W^{I}$ and $w^{2}$ are directly connected. Therefore we may express a hypothesis that it is sufficient to store same informations concerning the single word $W^{I}$ instead of the whole translation of $S_{1}$. 
In other words these informations concerning $w^{1}$ are the necessary context, when we want to translate conectly $\mathrm{S}_{2}$ 。 It is similar in other cases.

What concerns the translation of the particular sentences which are decomposed into the primitive phrases the main principales are described in $[4]$, because it is casy to indtroduced to each prinitive phrase a corresponding rule as in a phrase structer grammar[1]. 
[1] Chomsky $\mathrm{k}$, On certain formal properties of ercmmars. Infozwotion and Control 2(1959),137-167

[2] Culik $\mathrm{K}$, Some problems in theory of languaces (Czech), Proceedings of 1 .conference on cybernetics 1952 (in print)

[3] Culik K., ipplication of abstract semantics and thenry of graphs to polyglot dictioneries (Ruscien), t'roblems of Cybernetics (in print)

[4] Culik K., Semantics and Translation of Craminars and ALGOL-like langueges, Kybernetika 1(196j), 47-49

[5] Glushkoff V.in., Syntresis of di ittal automata (Russian), inosc ON 1962 